\title{
Colorectal cancer stem cell and chemoresistant colorectal cancer cell phenotypes and increased sensitivity to Notch pathway inhibitor
}

\author{
RUI HUANG ${ }^{1 *}$, GUIYU WANG $^{1 *}$, YANNI SONG ${ }^{2}$, QINGCHAO TANG $^{1}$, QI YOU $^{1}$, ZHENG LIU $^{1}$, \\ YINGGANG CHEN ${ }^{1}$, QIAN ZHANG ${ }^{1}$, JIAYING LI ${ }^{1}$, SHAN MUHAMMAND ${ }^{1}$ and XISHAN WANG ${ }^{1,3}$ \\ ${ }^{1}$ Department of Colorectal Surgery, The Second Affiliated Hospital of Harbin Medical University, Harbin, Heilongjiang 150086; \\ ${ }^{2}$ Department of Breast Surgery, The Third Affiliated Hospital of Harbin Medical University, Harbin, Heilongjiang 150081; \\ ${ }^{3}$ Colorectal Cancer Institute of Harbin Medical University, Harbin, Heilongjiang 150086, P.R. China
}

Received October 30, 2013; Accepted May 9, 2014

DOI: $10.3892 / \mathrm{mmr} .2015 .3694$

\begin{abstract}
Colorectal cancer stem cells (Co-CSCs) are a small subpopulation of tumor cells which have been proposed to be tumor-initiating cells in colorectal cancer (CRC) and to be implicated in resistance to standard chemotherapy. Chemoresistance is a common problem in the clinic. However, the interrelation between Co-CSCs and chemoresistant cells has yet to be elucidated. The present study investigated the Co-CSC phenotype in colonospheres and chemoresistant CRC cell lines and aimed to identify targets for therapy. Colonospheres and chemoresistant CRC cells were found to be enriched with the CSC markers CD133 and CD44, and exhibited similar phenotypes. Furthermore, it was found that Notch signaling may simultaneously regulate Co-CSCs and chemoresistant cells and may represent a novel strategy for targeting this pathway in CRC.
\end{abstract}

\section{Introduction}

Colorectal cancer (CRC) is a major cause of cancer mortality and morbidity worldwide. It is the third most commonly diagnosed cancer in males and the second in females, with $>1.2$ million new cases and 608,700 mortalities estimated to have occurred in 2008 (1). At present, the identification of novel strategies for therapy is urgently required.

Correspondence to: Dr Xishan Wang, Department of Colorectal Surgery, The Second Affiliated Hospital of Harbin Medical University, 246 Xuefu Road, Harbin, Heilongjiang 150086, P.R. China E-mail: wxshan1208@yahoo.com.cn

*Contributed equally

Key words: colorectal cancer, cancer stem cells, chemoresistant cells, Notch pathway, CD133/CD44
The colorectal cancer stem cell (Co-CSC) hypothesis provides a novel understanding of tumorigenesis. Increasing evidence shows that Co-CSC subpopulations are capable of self-renewal, driving tumor growth and differentiating to form all of the lineages observed within a tumor (2-5). Therefore, Co-CSCs may represent a novel target for the treatment of tumors (6). Current strategies for cancer treatment require modification to ensure that the Co-CSCs which are driving tumor growth are specifically targeted. However, there is increasing evidence that Co-CSCs are more resistant to current chemotherapy (7) than other subpopulations of cells within the tumor. This may be one of the reasons why the effectiveness of standard chemotherapy is limited. Standard chemotherapeutics are incapable of eradicating Co-CSCs, potentially due to drug efflux, autocrine survival signaling and alterations in DNA damage repair mechanisms in Co-CSCs $(8,9)$. However, the specific mechanism underlying Co-CSC chemoresistance has yet to be elucidated.

5-fluorouracil (5FU) and oxaliplatin are the predominant chemotherapeutic agents used for treating advanced CRC. $5 \mathrm{FU}$ and oxaliplatin have different mechanisms of action. $5 \mathrm{FU}$ inhibits the activity of the thymidylate synthase enzyme during DNA replication (10), while oxaliplatin causes prolonged $\mathrm{G}_{2}$-phase arrest and inhibits tumor cell growth through covalent DNA binding (11). Although the mechanisms of tumor cell resistance to 5FU and oxaliplatin have been extensively investigated, the specific mechanism remain to be fully elucidated. Previous studies have reported that these chemoresistant cells overexpress markers of CSCs (12). This shows that chemoresistant tumor cells represent a subpopulation of cells from the original tumor which are molecularly and phenotypically distinct. Therefore, the association between Co-CSCs and chemoresistant cells is of interest. Co-CSCs exhibit characteristics of cells which are resistant to standard chemotherapeutics and chemoresistant cells also express markers of Co-CSCs. Therefore, Co-CSCs and chemoresistant cells may be interrelated. Thus, the identification of a common target of Co-CSCs and chemoresistant cells may be of significance for clinical treatment.

A number of important signaling pathways, including Wnt, Notch and Hedgehog, have been found to have a role 
in regulating the self-renewal of CSCs in the hematopoietic system, skin, nervous system and breast (13-15). However, the mechanism by which pathways, including the Notch pathway, regulate Co-CSCs and chemoresistant cells, as well as the role that Notch has in the interrelation between these two types of cells, has yet to be elucidated.

Based on the clinical significance of chemoresistance and the ineffectiveness of chemotherapy in eliminating Co-CSCs, the present study aimed to investigate the interrelation between Co-CSCs and chemoresistant cells. Molecular and phenotypic alterations were investigated in Co-CSCs and chemoresistant cells in vitro, as well as using a mouse model system in vivo. Notch pathway activation was also detected in the Co-CSCs and chemoresistant cell lines and was targeted using xenograft models. The present study has two clinical implications associated with the interrelation between Co-CSCs and chemoresistant cells. The first is that it translates the theory of CSCs into clinical practice and shows that similar mechanisms may act in Co-CSCs and chemoresistant cells. The second is that it shows that Notch signaling simultaneously regulates Co-CSCs and chemoresistant cells and identifies a novel mechanism of targeting the Notch signaling pathway in CRC. Of note, the altered Notch activity observed in the present study may partially explain the chemoresistance in Co-CSCs and chemoresistant cells.

\section{Materials and methods}

Cell lines and culture. The HCT116 human CRC cell line was obtained from the Colorectal Cancer Institute of Harbin Medical University (Harbin, China). Colonospheres were cultured as described previously (16). In brief, using a limited dilution method, 1-3 cells were seeded on a 96-well ultralow-attachment plate (Corning Life Sciences, Corning, NY, USA) with Dulbecco's modified Eagle's medium (DMEM)/F12 medium containing B27 supplement (Invitrogen Life Technologies, Carlsbad, CA, USA), $20 \mathrm{ng} / \mathrm{ml}$ basic fibroblast growth factor and $20 \mathrm{ng} / \mathrm{ml}$ epidermal growth factor, which served as the stem cell medium (SCM) for the experiments. Under these culture conditions, Co-CSCs, but not differentiated cancer cells, are able to survive and proliferate (17-19). The number of surviving cells in each well of the 96-well plate was then observed and one cell well was selected and marked. After seven days, the cells were observed and the death cell well was removed and SCM was added to the survival cell well. After 10-14 days, the well in which colonospheres grew was marked and colonospheres were supplemented with SCM every three days until the colonospheres were able to be passaged.

An oxaliplatin-resistant cell line (HCT116/OxR) and 5FU-resistant cell line (HCT116/5FU-R) were developed as previously described $(20,21)$. The resistant cells and parental cells were grown in DMEM containing $10 \%$ fetal bovine serum (FBS; Sigma-Aldrich, St. Louis, MO, USA) and 1\% antibiotic solution (Mediatech Inc., Herndon, VA, USA) at $37^{\circ} \mathrm{C}$ in a humidified incubator containing $5 \% \mathrm{CO}_{2}$.

Drugs and antibodies. Oxaliplatin and 5FU were purchased from the Colorectal Cancer Institute of Harbin Medical University. The $\gamma$-secretase inhibitor $N$-[N-(3,5-difluoroph enacetyl)-L-alanyl]-S-phenylglycine $t$-butyl ester (DAPT) was purchased from Sigma-Aldrich and was used to inhibit Notch signaling in vitro and in vivo. The antibodies used for flow cytometry, immunohistochemistry (IHC), immunofluorescence and western blot analysis were as follows: Rabbit anti-cluster of differentiation (CD) 133, rabbit anti-Notch1 (Cell Signaling Technology, Inc., Beverly, MA, USA), rabbit anti- $\beta$-actin (Sigma-Aldrich), mouse anti-CD44 (Abcam PLC, Cambridge, UK), allophycocyanin (APC)-conjugated anti-CD133, APC-conjugated mouse-immunoglobulin G (IgG) 1 (Miltenyi Biotec, Bergisch Gladbach, Germany), phycoerythrin (PE)-conjugated anti-CD44, PE-conjugated mouse-IgG2b (Becton, Dickinson and Company, Franklin Lakes, NJ, USA), rabbit anti-hairy and enhancer of split-1 (HES-1; Santa Cruz Biotechnology, Inc., Santa Cruz, CA, USA) and mouse anti-Ki67 (Dako, Ely, UK).

Proliferation and chemosensitivity. Cell proliferation and drug sensitivity were assessed using a Cell Counting Kit-8 (CCK-8) assay as described previously (22). In brief, the cell lines were seeded at a density of 5,000 cells/well in 96-well plates in $100 \mu 1$ media with or without 5FU, oxaliplatin or DAPT. At each time-point $(0,24,48$ and $72 \mathrm{~h}), 10 \mu \mathrm{l}$ CCK-8 solution was added to each well and incubated for a further $2 \mathrm{~h}$. The absorbance was read at $450 \mathrm{~nm}$ using a standard microplate reader.

Colonosphere assay. Each cell line was trypsinized and quantified by plating a single cell in each well of a low-attachment 96-well plate and counted under a microscope, assessing the rate of colonospheres. The colonospheres were cultured using the aformentioned process.

Clonogenic assay. Clonogenic assays were performed to determine the proliferative capacity of the colonospheres and the chemoresistant cells. A total of 500 cells/well were seeded on a six-well plate and incubated for 14 days at $37^{\circ} \mathrm{C}$ in $5 \%$ $\mathrm{CO}_{2}$. Following incubation, the colonies were formalin-fixed and stained with hematoxylin. Colonies which were $>50 \mu \mathrm{m}$ were counted under a light microscope (CKX31; Olympus, Tokyo, Japan) and compared with the parental cells.

Western blot analysis. Cell lysates were subjected to SDS-PAGE and blotted onto Immobilon ${ }^{\circledR}$-P polyvinylidene difluoride membranes (Millipore Corporation, Billerica, MA, USA). Specific proteins were detected using an enhanced chemiluminescence system (GE Healthcare, Little Chalfont, UK). Membranes were probed with the aforementioned antibodies.

Flow cytometry. Single cells were prepared for the analysis of cell surface markers by digesting with pancreatin. Cells were then detached from the plates through incubation with enzyme-free cell dissociation buffer (Invitrogen Life Technologies). Cells were then washed with $10 \mathrm{mmol} / 1$ cold phosphate-buffered saline (PBS) and resuspended in $1 \mathrm{X}$ binding buffer (BD Biosciences, San Jose, CA, USA) at a concentration of $1 \times 10^{6}$ cells $/ \mathrm{ml}$. Cells were subjected to direct immunofluorescence staining using APC-conjugated anti-CD133 and PE-conjugated anti-CD44 antibodies followed by flow cytometric analysis. Samples were analyzed using a 
NucleoCounter ${ }^{\circledR}$ NC-3000 analyzer (ChemoMetec, Lillerød, Denmark). The experiments were repeated at least three times.

In vivo assay and Notch pathway inhibition. Male nude mice, aged four weeks, were purchased from the Shanghai Laboratory Animal Center (Shanghai, China). All animal experiments were approved by the Institutional Animal Care and Use Committee of Harbin Medical University. Equal numbers of cells $\left(1 \times 10^{6}\right)$ from colonospheres and chemoresistant cell lines were suspended in $100 \mu 1 \mathrm{PBS}$ and injected subcutaneously into the flank of each mouse (six mice per group). When the tumors reached $\sim 100 \mathrm{~mm}^{3}$, mice were subjected to intraperitoneal injection with $200 \mu \mathrm{g}$ DAPT twice per week. Tumor growth was observed and recorded over 10 weeks. When the tumors in the control group exceeded $1.5 \mathrm{~cm}$ in diameter, the animals were euthanized and the tumors were weighed and measured. Tumor volume was calculated using the following formula: (length)/2x(width) ${ }^{2}$. Tumors were then paraffin-embedded, sectioned and stained with hematoxylin and eosin (H\&E) for histological analysis. IHC and apoptotic analysis were also performed. Data are shown from representative experiments.

Immunohistochemistry and immunofluorescent analysis. IHC was performed on 4- $\mu \mathrm{m}$ sections which were prepared using the paraffin-embedded tissues. Following standard procedures, tissues were deparaffinized using xylene, hydrated in graded alcohol and pretreated for antigen retrieval in Tris/ethylene diaminetetracetic acid buffer for $5 \mathrm{~min}$ in a $100^{\circ} \mathrm{C}$ steamer. Slides were then H\&E stained in order to assess morphology or incubated with anti-Ki67 antibodies to visualize the proliferative nuclei. All sections were developed using 3,3'-diaminobenzidine and counterstained with hematoxylin (Invitrogen Life Technologies). Apoptotic cells within the tissue were detected using the terminal deoxynucleotidyltransferase-mediated dUTP nick-end labeling (TUNEL) assay. An Apoptag in situ apoptosis detection kit (Roche Diagnostics, Mannheim, Germany) was used for TUNEL staining according to the manufacturer's instructions for paraffin-embedded tissues. Immunohistochemical staining and fluorescence were analyzed using a Zeiss Axioskop microscope (Carl Zeiss AG, Oberkochen, Germany) and apoptosis was expressed as the percentage of TUNEL positive cells.

Statistical analysis. All data are presented as the mean \pm standard error of three independent experiments, each performed in triplicate. Data were analyzed using the Student's t-test. Analysis of variance was performed for multiple comparisons. $\mathrm{P}<0.05$ was considered to indicate a statistically significant difference. SPSS 17.0 statistical software (SPSS, Inc., Chicago, IL, USA) was used for the analyses.

\section{Results}

Expression of CSC markers in the colonospheres and chemoresistant cells. CRC has been proposed to arise specifically in stem cell populations at the base of colonic crypts. Markers used for the identification of Co-CSCs include CD44, CD133, CD24, CD29, leucine-rich repeat-containing G-protein coupled receptor 5 and doublecortin-like kinase 1 (23). Among these markers, CD44 and CD133 have been widely used for the identification of CSCs in CRC. The CSC population has been reported to be capable of self-renewal and generating tumors resembling the primary tumor. Moreover, CSCs have been found to be capable of growth in serum-free medium and the formation colonospheres. In the present study, the expression profiles of HCT116 human CRC colonospheres and cells resistant to $5 \mathrm{FU}$ or oxaliplatin (HCT116/5FU-R or HCT116/OxR, respectively) were assessed using western blot analysis and flow cytometry. Compared with the parental HCT116 cells, CD133 and CD44 expression were observed to be significantly higher in the colonospheres, HCT116/5FU-R and HCT116/OxR cells (Fig. 1A). The number of cells expressing CD133 and CD44 was also found to be significantly higher in the colonospheres and chemoresistant cells compared with the parental cells (Fig.1B), with only $2 \%$ of the parental cells expressing CD133 and $48 \%$ expressing CD44, while between 33 and $65 \%$ of the three cell types expressed CD133, and between 84 and 93\% of the three cell types expressed CD44. Following CD133 and CD44 labeling, flow cytometric analysis revealed a 4.8 -fold enrichment of $\mathrm{CD} 133^{+} / \mathrm{CD} 44^{+}$cells in the HCT116/5FU-R cell line, a 22 -fold enrichment of $\mathrm{CD} 133^{+} / \mathrm{CD} 44^{+}$cells in the oxaliplatin-resistant cell line and a 24.7 -fold enrichment of $\mathrm{CD} 133^{+} / \mathrm{CD}_{4} 4^{+}$cells in the colonospheres compared with the parental HCT116 cells (Fig. 1C).

Cell phenotype in the colonospheres and chemoresistant cells. In vitro proliferation was assessed through plating an equal number of cells from each cell line and using a CCK-8 assay as an index of cell number. The proliferation rates of the colonospheres, 5FU- and oxaliplatin-resistant cells were found to be significantly lower than those of the parental cells (52-72\%; P<0.05; Fig. 2A). The CCK-8 assay was also used to analyze cell sensitivity to chemotherapeutic agents. Colonospheres, 5FU- and oxaliplatin-resistant cells were exposed to clinically relevant doses of $5 \mathrm{FU}$ and oxaliplatin. The number of cells remaining after $72 \mathrm{~h}$ was then assessed. Parental cells were found to be sensitive to oxaliplatin and $5 \mathrm{FU}$, with only 34 and $21 \%$ of the cells remaining viable following exposure to oxaliplatin and 5FU, respectively (Fig. 2B). 5FU-resistant cells were observed to be resistant to $5 \mathrm{FU}$; however, these cells were also resistant to oxaliplatin, with $77 \%$ of the cells remaining after $72 \mathrm{~h}$ of exposure. Similarly, oxaliplatin-resistant cells were found to be resistant to oxaliplatin, but also exhibited cross-resistance to $5 \mathrm{FU}$. Colonospheres were resistant to oxaliplatin and 5FU, with $79-87 \%$ of the cells remaining viable after $72 \mathrm{~h}$ of exposure.

CSCs have the capacity to form colonies, also known as spheres, in the absence of serum and without attachment to culture plates. In the present study, the capacity of colonospheres and chemoresistant cell lines to grow colonospheres under serum-free conditions was analyzed. Cell lines were trypsinized and quantified by plating a single cell in each well of a low-attachment 96-well plate and assessing the capacity of the cells to form colonospheres. In the colonosphere cells, the rate of secondary sphere generation was higher than that in the HCT116/5FU-R and HCT116/OxR cells. However, compared with the parental cells, an increased number of colonospheres was found in the three cell types $(\mathrm{P}<0.05$; Fig. 2C). The clonogenic assay revealed that the three types of 
A

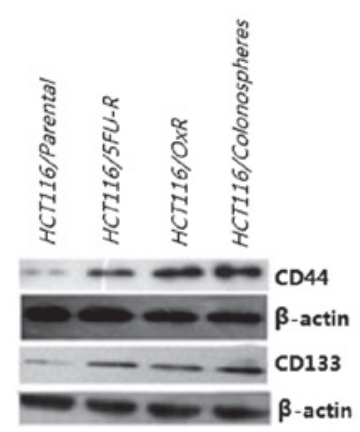

C

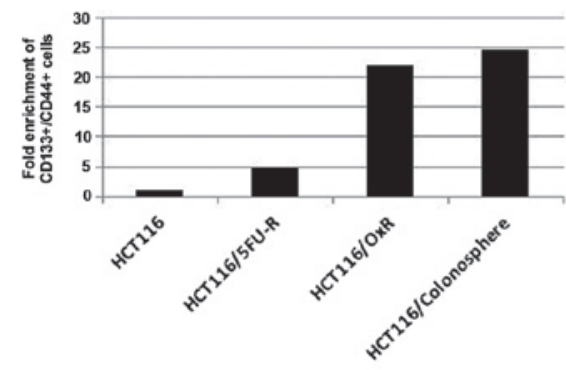

B
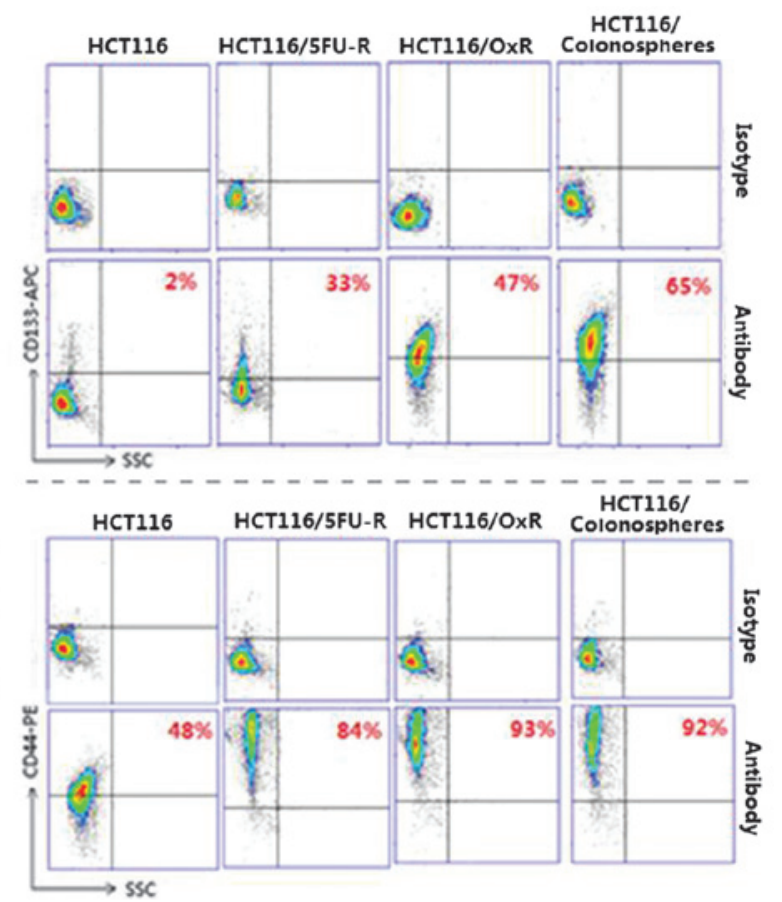

Figure 1. Colonospheres and chemoresistant cell lines are enriched with Co-CSC markers. (A) Western blot analysis revealed that expression of the Co-CSC markers CD133 and CD44 was higher in the colonospheres and HCT116/5FU-R and HCT116/OxR chemoresistant cells compared with the parental HCT116 human CRC cells. $\beta$-actin was used as a loading control. (B) Flow cytometric analysis revealed that the colonospheres and chemoresistant cell lines were enriched with cells expressing CD133 and CD44 compared with the parental cell line. A total of 33\% of the HCT116/5FU-R cells, 47\% of the HCT116/OxR cells and $65 \%$ of the HCT116/colonosphere cells expressed CD133 compared with $2 \%$ of the parental HCT116 cells. Similarly, $84 \%$ of the HCT116/5FU-R cells, $93 \%$ of the chemoresistant cells and $92 \%$ of the HCT116/colonosphere cells expressed CD44 compared with $48 \%$ of the parental cells. Cytometric analysis plots using isotype control antibodies were used as staining controls. (C) CD44 and CD133 labelling and flow cytometric analysis revealed a 4.8-, 22- and 24.7-fold enrichment of double-positive cells in the HCT116/5FU-R, HCT116/OxR and colonosphere cells compared with the parental HCT116 cell line. SCC, side scatter; Co-CSC, colorectal cancer stem cell; CD, cluster of differentiation; 5-FU, 5-fluorouracil; R, resistant; Ox, oxaliplatin.

cells exhibited an increased colonosphere formation capacity after 14 days of culture (Fig. 2D).

Notch pathway in colonospheres and chemoresistant cells. Western blot analysis was used to assess constitutive signaling in the parental, colonosphere and chemoresistant cell lines, with a focus on targets for which agents that inhibited target function were readily available. Several signaling pathways were investigated, but the most marked alterations were observed in the Notch signaling pathway, thus the present study focused on Notch signaling. Notch1 levels were found to be higher in the colonospheres and chemoresistant cell lines compared with the parental cells (Fig. 3A). Furthermore, the levels of hairy and enhancer of split 1 (Hes1) were also observed to be increased in the colonospheres and chemoresistant cell lines compared with the parental cells.

DAPT, a $\gamma$-secretase inhibitor, was used to determine the dependence of the cells on Notch signaling for survival. The CCK-8 assay revealed that DAPT treatment caused a minor decrease $(12 \%)$ in cell number in the parental cells, but a significantly greater reduction in cell number in the colonospheres and chemoresistant cells compared with the parental cells (42\% for HCT116/5FU-R, 48\% for HCT116/OxR and $51 \%$ for HCT116/colonospheres; all $\mathrm{P}<0.05$; Fig. 3B).

Effect of Notch pathway inhibition on in vivo tumor growth. Colonospheres and chemoresistant cells were injected subcutaneously in the flanks of the nude mice and tumor growth was assessed during biweekly treatment with DAPT or dimethyl sulfoxide (DMSO). After four weeks, at which point the maximum tumor size was $\sim 1.5 \mathrm{~cm}^{3}$, the tumors were harvested and analyzed. The tumors derived from the colonospheres, parental and chemoresistant cells which were treated with DAPT were found to be significantly smaller than those treated with DMSO (control). However, the tumors derived from the colonospheres and chemoresistant cells exhibited significantly greater DAPT-induced growth inhibition compared with the parental cells. The 5FU-resistant and oxaliplatin-resistant cells showed 52 and $67 \%$ growth inhibition, respectively, while growth of the colonosphere cells was inhibited by $71 \%$ compared with the parental cells (21\%; $\mathrm{P}<0.05$; Fig. 4A).

Analysis of the proliferation marker Ki67 using tumor section staining revealed that DAPT caused a decrease in the number of proliferating cells in all of the tumors compared with those treated with DMSO. Similar to tumor growth inhibition, the inhibition of Notch signaling had a greater effect on the tumors derived from the colonospheres and chemoresistant cells than on the tumors derived from the parental cells; however, this difference was not found to be statistically significant. TUNEL staining was used to analyze apoptosis in the xenografts. Quantification of TUNEL staining showed that DAPT treatment caused significantly more apoptosis in the tumors derived from the colonospheres and chemoresistant cells compared with those derived from the HCT116 cells $(\mathrm{P}<0.05)$. Specifically, upon Notch signaling inhibition, HCT116 tumors showed a 2.1-fold increase in apoptotic 
A

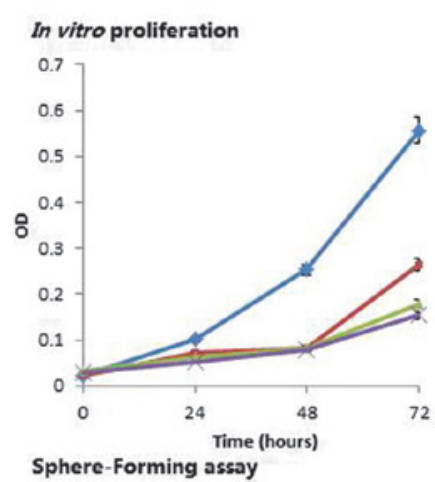

C

D

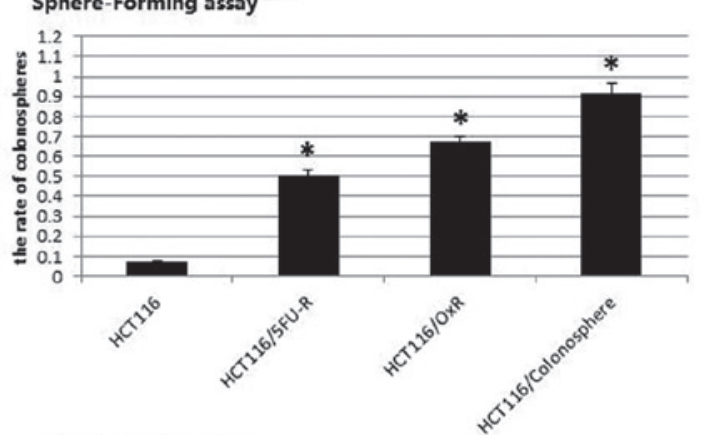

B
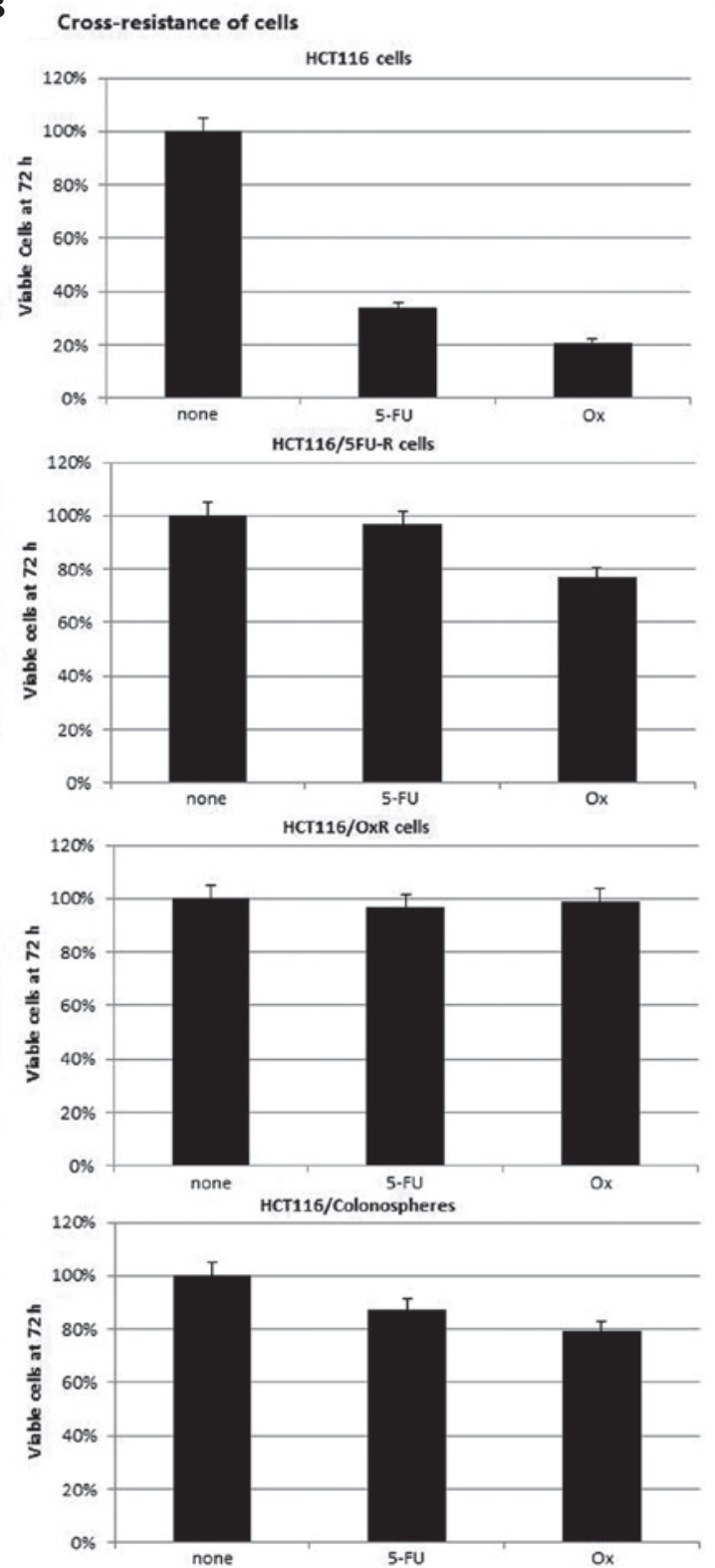

Figure 2. Colonospheres and chemoresistant cell exhibit a cancer stem cell phenotype. (A) Colonospheres and chemoresistant cells proliferated at a significantly slower rate than parental cells, detected using the Cell Counting Kit-8 assay. (B) Parental cells were sensitive to 5FU and Ox following exposure for $72 \mathrm{~h}$, with only 34 and $21 \%$ of cells remaining, respectively, compared with the untreated cells. By contrast, HCT116/5FU-R cells were resistant to $5 \mathrm{FU}$; however, they also exhibited increased resistance to Ox compared with the parental cells. Similarly, HCT116/Ox-R cells were resistant to Ox, as well as 5FU. Colonosphere cells were also resistant to Ox and 5-FU. (C) Cells were plated in an ultralow-attachment 96-well plate in the absence of serum and after 14 days, the rate of viable sphere-forming cells was assessed. The colonosphere formation rate was significantly increased in the colonospheres and chemoresistant cells compared with the parental cells. (D) Clonogenic assay revealed that the number of colonies larger than $50 \mu \mathrm{m}$ in diameter which were formed under standard growth conditions was significantly higher in the colonospheres and chemoresistant compared with the parental HCT116 cells. Data are presented as the mean \pm standard error. ${ }^{*} \mathrm{P}<0.05$ vs. HCT116 cells. OD, optical density, 5-FU, 5 -fluorouracil; Ox, oxaliplatin; R, resistant.

nuclei compared with 5.4-, 5.9- and 5.7-fold increases in the HCT116/5FU-, HCT116/OxR- and colonosphere-derived tumors, respectively (all $\mathrm{P}<0.05$; Fig. 4B). Representative images from the analyzed tumor sections and the subcutaneous tumors are shown in Fig. 5.

\section{Discussion}

CRC is the second leading cause of cancer-associated mortality worldwide. Despite recent therapeutic regimens which have markedly increased survival in CRC, almost all CRC tumors become chemoresistant (24). Thus, it is necessary to understand the mechanisms of resistance in order to improve current treatment protocols in CRC.

The eradication of drug-resistant Co-CSCs is an important area of investigation (25). Numerous studies have used fluorescence-activated cell sorting (FACS) in order to identify and isolate CSCs. In the present study, FACS was not performed on CSCs as no reliable surface markers are available for identifying Co-CSCs. However, CSCs have the capacity to form colonies, also know as spheres, when cultured in the absence of serum. Therefore, colonosphere formation was used to investigate 
A

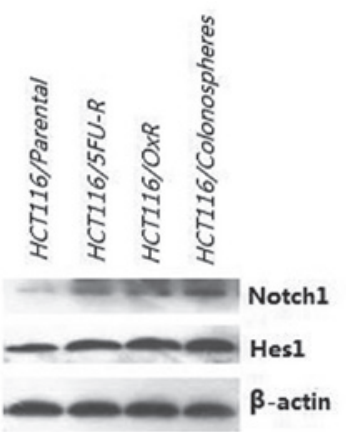

B

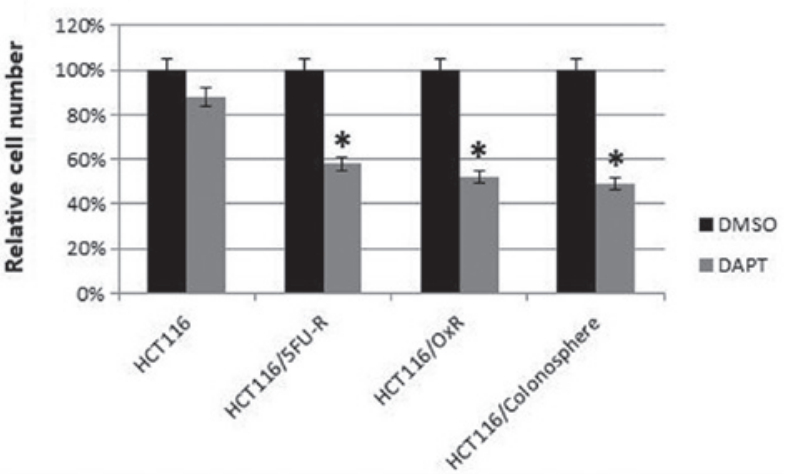

Figure 3. Effect of Notch pathway inhibition on colonospheres and chemoresistant cells. (A) Analysis of whole-cell lysates from parental HCT116 cells, chemoresistant HCT116/5FU-R and HCT116/Ox-R cells and colonosphere cells revealed an increase in Notch1 and Hes1 in the chemoresistant and colonosphere cells compared with the parental cells. (B) Cells were treated with DMSO (control) or a Notch pathway inhibitor DAPT and the cell number was assessed. Upon DAPT treatment, the cell number was significantly decreased in the chemoresistant and colonosphere cells (42-51\%) compared with the HCT116 cells (12\%). ${ }^{*} \mathrm{P}<0.05$ vs. HCT116 cells. 5-FU, 5-fluorouracil; R, resistant; Ox, oxaliplatin; DAPT, $N$-[N-(3,5-difluorophenacetyl)-L-alanyl $]-S$-phen ylglycine $t$-butyl ester; DMSO, dimethyl sulfoxide; Hes, hairy and enhancer of split.

the characteristics of Co-CSCs (26). Chemoresistance is an important feature of Co-CSCs, thus the present study aimed to investigate the interrelation between Co-CSCs and chemoresistant cells. The present study focused on colonospheres and two chemoresistant cell lines and identified certain common features. Colonospheres and chemoresistant cells were found to be significantly enriched in the CSC markers CD133 and CD44 (27). This finding suggested that colonospheres may be abundant in Co-CSCs. Furthermore, the chemoresistance imparted in the two chemoresistant cell lines may be due to the acquisition of CSC phenotypes in these cell lines. Thus, in the present study, to further investigate the characteristics of Co-CSCs, colonospheres were cultured in serum-free media and chemoresistant cell lines were developed.

Colonospheres and chemoresistant cells were also found to be more quiescent in vitro, with decreased cell proliferation compared with the parental cells. However, a clonogenic assay revealed that colonospheres and chemoresistant cells had an increased capacity to form colonies and spheres in specialized serum-free media, characteristics which are consistent with the CSC phenotype (5). Furthermore, lysates obtained from cell line-derived colonospheres were observed to have increased resistance to 5FU and oxaliplatin as compared with the adherent parental cells. Oxaliplatin-resistant cells and 5FU-resistant cells also exhibited cross-resistance to 5FU and oxaliplatin,
A

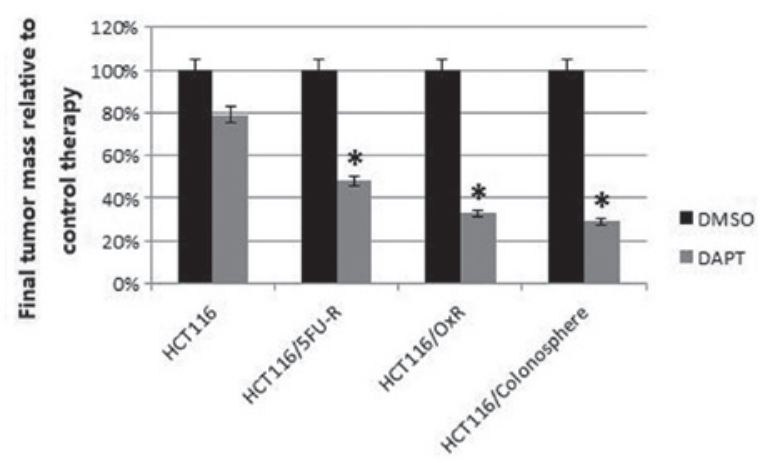

B

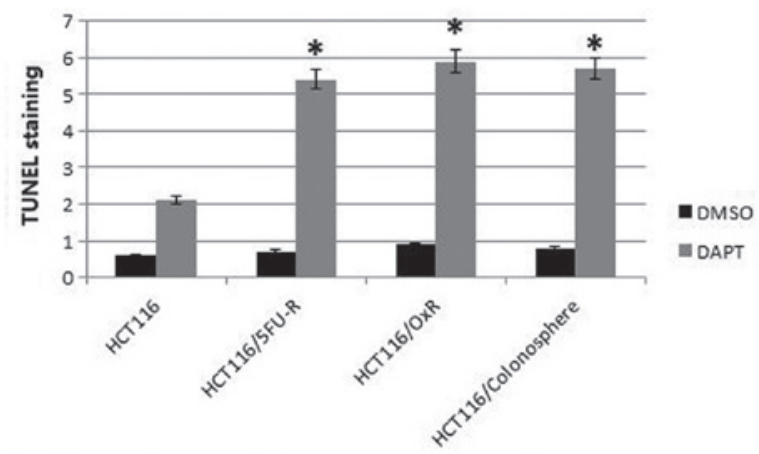

Figure 4. Effect of Notch pathway inhibition on in vivo tumor growth, proliferation and apoptosis. Mice were subcutaneously injected with $1 \times 10^{6}$ HCT116, HCT116/5FU-R, HCT116/OxR or HCT116/colonosphere cells and treated with DMSO (control) or DAPT twice weekly. Final tumor masses were measured and compared between mice bearing tumors from each cell line. (A) In the DAPT-treated mice, the HCT116/5FU-R-, HCT116/OxR- and HCT116/colonosphere-derived tumors showed significantly greater growth inhibition than the HCT116-derived tumors. (B) In the DAPT-treated mice, TUNEL staining revealed significantly greater apoptosis in the HCT116/5FU-R-, HCT116/OxR- and HCT116/colonospheres-derived tumors than in tumors derived from the HCT116 cells. Data are presented as the mean \pm standard error. ${ }^{*} \mathrm{P}<0.05$ vs. HCT116 cells. 5-FU, 5-fluorouracil; R, resistant; Ox, oxaliplatin; DAPT, $N$-[N-(3,5-difluorophenacetyl)-L-alanyl]-S -phenylglycine $t$-butyl ester; DMSO, dimethyl sulfoxide; TUNEL, terminal deoxynucleotidyltransferase-mediated dUTP nick-end labeling.

respectively. This suggested that colonospheres and chemoresistant cells activated general resistance pathways leading to multi-drug resistance. Colonospheres and chemoresistant cells were also found to be enriched in CSC markers and properties, consistent with the CSC phenotype. It is most likely that the process of developing colonospheres and chemoresistant cell lines involved increasing the expression of such CSC markers, rather than enriching the population of cells which already expressed these markers. Assessing this hypothesis may be difficult; however, previous studies have shown that the tumor microenvironment, including soluble factors and hypoxia, may affect colonosphere characteristics $(28,29)$. However, little is known about the pathway involved in the expression of CSC markers in Co-CSCs and chemoresistant cells and further investigations into the mechanism of resistance and increased marker expression are required.

Therefore, the present study aimed to investigate the pathways involved in CSCs and chemoresistant cells and aimed to identify potential targets in the colonospheres and chemoresistant cells which would allow specific targeting of these cells with optimal agents. One such pathway was the Notch signaling pathway, which is involved in CRC 

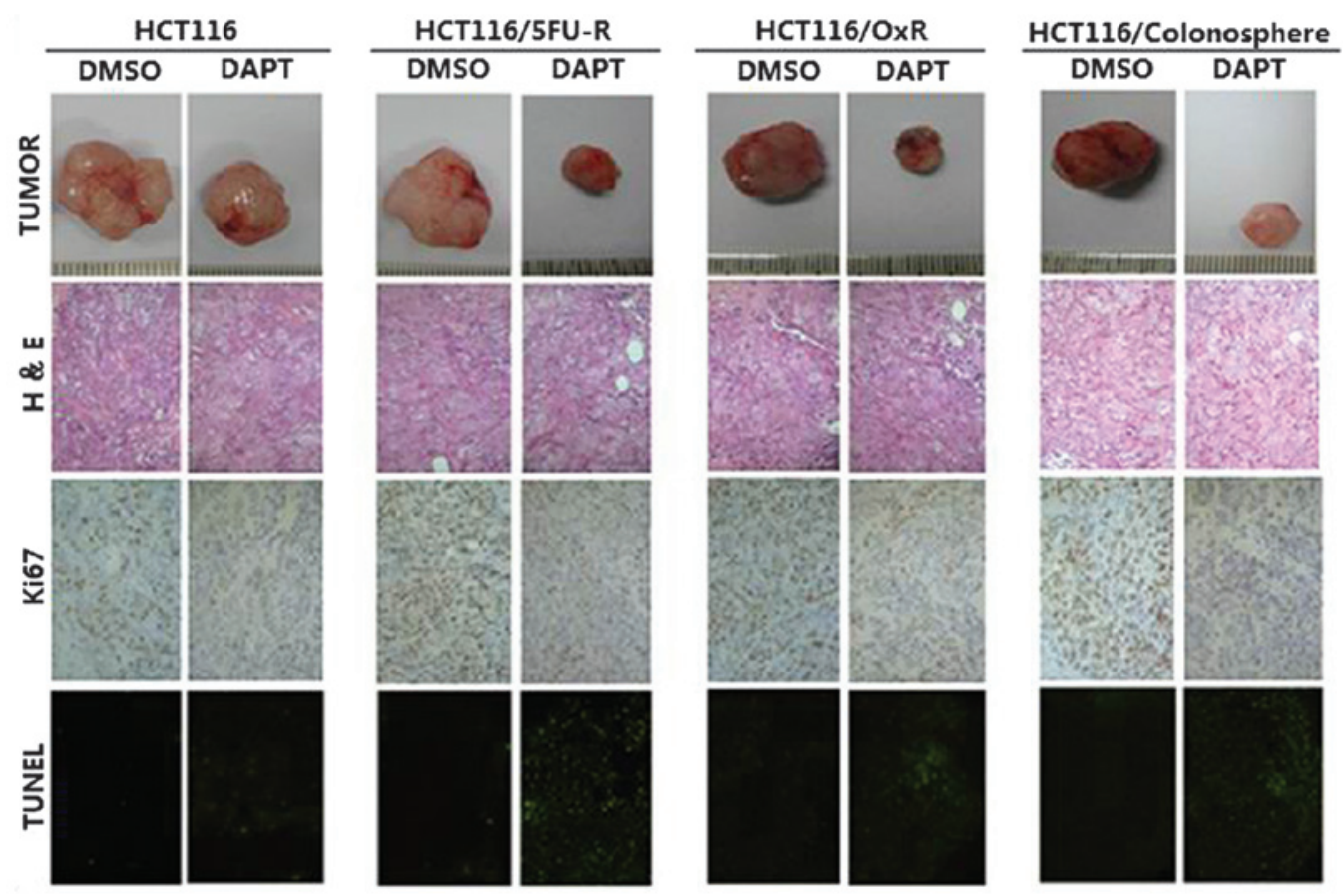

Figure 5. Effect of Notch pathway inhibition on in vivo tumor characteristics. Immunohistochemical analysis of tumors was performed and multiple tumor fields were analyzed per group. Representative images of all groups and treatments are presented (magnification, x100). H\&E staining revealed similar subcutaneous tumor morphology among all groups of tumors. Ki67 staining showed decreased cell proliferation in the tumors treated with DAPT; however, no significant differences were observed in the cell proliferation between the HCT116-, colonosphere-, HCT116/5FU-R- and HCT116/Ox-R-derived tumor sections. TUNEL staining revealed significantly increased apoptosis in response to DAPT in colonosphere-, HCT116/5FU-R-and HCT116/Ox-R-derived tumors compared with tumors derived from HCT116 cells (P<0.05). 5-FU, 5-fluorouracil; R, resistant; Ox, oxaliplatin; DAPT, $N$-[ $N$-(3,5-difluorophenacetyl)-L-alanyl]-S-phenylglycine $t$-butyl ester; DMSO, dimethyl sulfoxide; TUNEL, terminal deoxynucleotidyltransferase-mediated dUTP nick-end labeling; H\&E, hematoxylin and eosin.

progression and growth $(30,31)$. The Notch1 gene copy number has been reported to be increased in colorectal adenocarcinomas and to be correlated with aggressive tumor behavior and poor prognosis (32-34). In the present study, constitutive Notch1 expression was observed to be higher in the colonospheres and chemoresistant cell lines compared with the parental cell lines, and most markedly increased in the colonospheres. Furthermore, the increase in Hes1 was associated with an increase in the levels of the targeted gene. In the present study, the Notch signaling pathway, particularly Notch1, was found to have a key role in colonospheres and chemoresistant cell lines, and it was hypothesized that the activation of Notch was involved in maintaining a phenotypic characteristic in the colonospheres and chemoresistant cell lines. However, the specific mechanism underlying the increase in the Notch1 expression in these cells has yet to be elucidated. The inhibition of Notch signaling in vitro was found to cause a decrease in cell growth, as determined by CCK- 8 assay, and these effects were observed to be greater in the colonospheres and chemoresistant cell lines than in the parental cells. These findings showed that the inhibition of the Notch pathway using DAPT significantly depleted the number of colonosphere cells and chemoresistant cells, further validating the hypothesis that the activation of Notch is necessary for the maintenance of phenotypic characteristics in colonospheres and chemoresistant cell lines. In vivo, following DAPT treatment, the inhibition of the growth of colonosphere- and chemoresistant cell-derived tumors was found to be significantly higher than that of the parental cell-derived tumors. This finding suggested that the Notch signaling pathway may be important for Co-CSC maintenance and tumor resistance to standard chemotherapeutics. A previous study reported that colonic cancer cells may upregulate Notch1 as a protective mechanism in response to chemotherapy (35). Furthermore, in the present study, Notch inhibition was found to have a greater effect on the growth of colonosphere- and chemoresistant cell-derived tumors than parental cell-derived tumors in vivo, which was largely due to an increase in apoptosis. It is unlikely that colonospheres and chemoresistant cells acquired common molecular alterations to resist certain agents. However, the present study found that the colonospheres and chemoresistant cells acquired similar molecular and phenotypic alterations when cultured in serum-free media or when chronically exposed to chemotherapeutic agents. Of note, the colonospheres and chemoresistant cells also exhibited increased Notch1 and Hes1 expression, which led to these cells becoming more sensitive to the inhibition of the Notch pathway. Previous studies have suggested that targeting the Notch signaling pathway may be an effective method for targeting CSCs and chemoresistant cells $(36,37)$. The findings of the present study suggest that inhibiting the Notch pathway using DAPT may be an effective strategy for targeting Co-CSCs and overcoming the chemoresistance of CRC cells in a clinical setting.

\section{Acknowledgements}

The authors would like to thank Qiang Li for his helpful advice. 


\section{References}

1. Jemal A, Bray F, Center MM, Ferlay J, Ward E and Forman D: Global cancer statistics. CA Cancer J Clin 61: 69-90, 2011.

2. Reya T, Morrison SJ, Clarke MF and Weissman IL: Stem cells, cancer, and cancer stem cells. Nature 414: 105-111, 2001.

3. O'Brien CA, Pollett A, Gallinger S and Dick JE: A human colon cancer cell capable of initiating tumour growth in immunodeficient mice. Nature 445: 106-110, 2007.

4. Ricci-Vitiani L, Lombardi DG, Pilozzi E, et al: Identification and expansion of human colon-cancer-initiating cells. Nature 445: 111-115, 2007.

5. Yeung TM and Mortensen NJ: Colorectal cancer stem cells. Dis Colon Rectum 52: 1788-1796, 2009.

6. Sugihara E and Saya H: Complexity of cancer stem cells. Int J Cancer 132: 1249-1259, 2013.

7. Lou H and Dean M: Targeted therapy for cancer stem cells: the patched pathway and ABC transporters. Oncogene 26: 1357-1360, 2007.

8. Sussman RT, Ricci MS, Hart LS, Sun SY and El-Deiry WS: Chemotherapy-resistant side-population of colon cancer cells has a higher sensitivity to TRAIL than the non-SP, a higher expression of c-Myc and TRAIL-receptor DR4. Cancer Biol Ther 6: 1490-1495, 2007.

9. Todaro M, Alea MP, Di Stefano AB, et al: Colon cancer stem cells dictate tumor growth and resist cell death by production of interleukin-4. Cell Stem Cell 1: 389-402, 2007.

10. Walko CM and Lindley C: Capecitabine: a review. Clin Ther 27: 23-44, 2005

11. Kelland L: The resurgence of platinum-based cancer chemotherapy. Nat Rev Cancer 7: 573-584, 2007.

12. Wen K, Fu Z, Wu X, et al: Oct-4 is required for an antiapoptotic behavior of chemoresistant colorectal cancer cells enriched for cancer stem cells: effects associated with STAT3/Survivin. Cancer Lett 333: 56-65, 2013.

13. Holland JD, Klaus A, Garratt AN and Birchmeier W: Wnt signaling in stem and cancer stem cells. Curr Opin Cell Biol 25: 254-264, 2013.

14. Pannuti A, Foreman K, Rizzo P, et al: Targeting Notch to target cancer stem cells. Clin Cancer Res 16: 3141-3152, 2010.

15. Takebe N, Harris PJ, Warren RQ and Ivy SP: Targeting cancer stem cells by inhibiting Wnt, Notch, and Hedgehog pathways. Nat Rev Clin Oncol 8: 97-106, 2011.

16. Lee J, Kotliarova S, Kotliarov Y, et al: Tumor stem cells derived from glioblastomas cultured in bFGF and EGF more closely mirror the phenotype and genotype of primary tumors than do serum-cultured cell lines. Cancer Cell 9: 391-403, 2006.

17. Singh SK, Clarke ID, Terasaki M, et al: Identification of a cancer stem cell in human brain tumors. Cancer Res 63: 5821-5828, 2003.

18. Liu JC, Deng T, Lehal RS, Kim J and Zacksenhaus E: Identification of tumorsphere- and tumor-initiating cells in HER2/Neu-induced mammary tumors. Cancer Res 67: 8671-8681, 2007.

19. Vlashi E, Kim K, Lagadec C, et al: In vivo imaging, tracking, and targeting of cancer stem cells. J Natl Cancer Inst 101: 350-359, 2009 .
20. Yang AD, Fan F, Camp ER, et al: Chronic oxaliplatin resistance induces epithelial-to-mesenchymal transition in colorectal cancer cell lines. Clin Cancer Res 12: 4147-4153, 2006.

21. Dallas NA, Xia L, Fan F, et al: Chemoresistant colorectal cancer cells, the cancer stem cell phenotype, and increased sensitivity to insulin-like growth factor-I receptor inhibition. Cancer Res 69: 1951-1957, 2009.

22. Chu ZH, Liu L, Zheng CX, et al: Proteomic analysis identifies translationally controlled tumor protein as a mediator of phosphatase of regenerating liver-3-promoted proliferation, migration and invasion in human colon cancer cells. Chin Med J (Engl) 124: 3778-3785, 2011.

23. Vaiopoulos AG, Kostakis ID, Koutsilieris M and Papavassiliou AG: Colorectal cancer stem cells. Stem Cells 30: 363-371, 2012.

24. Marin JJ, Sanchez de Medina F, Castaño B, et al: Chemoprevention, chemotherapy, and chemoresistance in colorectal cancer. Drug Metab Rev 44: 148-172, 2012.

25. Puglisi MA, Tesori V,Lattanzi W, Gasbarrini GB and Gasbarrini A: Colon cancer stem cells: controversies and perspectives. World J Gastroenterol 19: 2997-3006, 2013.

26. Tirino V, Desiderio V, Paino F, et al: Cancer stem cells in solid tumors: an overview and new approaches for their isolation and characterization. FASEB J 27: 13-24, 2013.

27. Yu Y, Kanwar SS, Patel BB, et al: Elimination of colon cancer stem-like cells by the combination of curcumin and FOLFOX. Transl Oncol 2: 321-328, 2009.

28. Bose D, Zimmerman LJ, Pierobon M, et al: Chemoresistant colorectal cancer cells and cancer stem cells mediate growth and survival of bystander cells. Br J Cancer 105: 1759-1767, 2011.

29. Yeung TM, Gandhi SC and Bodmer WF: Hypoxia and lineage specification of cell line-derived colorectal cancer stem cells. Proc Natl Acad Sci USA 108: 4382-4387, 2011.

30. Koch U and Radtke F: Notch and cancer: a double-edged sword. Cell Mol Life Sci 64: 2746-2762, 2007.

31. Qiao L and Wong BC: Role of notch signaling in colorectal cancer. Carcinogenesis 30: 1979-1986, 2009.

32. Arcaroli JJ, Powell RW, Varella-Garcia M, et al: ALDH+ tumor-initiating cells exhibiting gain in NOTCH1 gene copy number have enhanced regrowth sensitivity to a $\gamma$-secretase inhibitor and irinotecan in colorectal cancer. Mol Oncol 6: 370-381, 2012.

33. Zhang Y, Li B, Ji ZZ and Zheng PS: Notch1 regulates the growth of human colon cancers. Cancer 116: 5207-5218, 2010.

34. Jin HY, Zhang HY, Wang X, Xu J and Ding Y: Expression and clinical significance of notch signaling genes in colorectal cancer. Tumour Biol 33: 817-824, 2012.

35. Meng RD, Shelton CC, Li YM, et al: gamma-Secretase inhibitors abrogate oxaliplatin-induced activation of the Notch-1 signaling pathway in colon cancer cells resulting in enhanced chemosensitivity. Cancer Res 69: 573-582, 2009.

36. McAuliffe SM, Morgan SL, Wyant GA, et al: Targeting Notch, a key pathway for ovarian cancer stem cells, sensitizes tumors to platinum therapy. Proc Natl Acad Sci USA 109: E2939-E2948, 2012.

37. Ponnurangam S, Mammen JM, Ramalingam S, et al: Honokiol in combination with radiation targets notch signaling to inhibit colon cancer stem cells. Mol Cancer Ther 11: 963-972, 2012. 